\title{
POSSÍVEL IMPACTO DA “LEI SECA" NOS ATENDIMENTOS A VÍTIMAS DE ACIDENTES DE TRÂNSITO EM UMA UNIDADE DE EMERGÊNCIA
}

\author{
Possible impact by the "dry law" (prohibition law) in attendance to victims of traffic \\ accidents in an emergency unit \\ Posible impacto de la "ley seca" en la asistencia a las víctimas de los accidentes de \\ tránsito en una unidad de emergencia
}

Ana Paula Pereira de Oliveira ${ }^{1} \quad$ Ângela Maria Mendes Abreu $^{2}$

Vanessa Silveira Faria ${ }^{4}$

\section{RESUMO}

Estudo quantitativo descritivo, cujos objetivos foram: determinar o número de indivíduos vítimas de acidentes de trânsito atendidos em um setor de emergência de um hospital municipal do Rio de Janeiro antes e depois da implementação da "Lei Seca" e identificar o perfil dessa população e as características dos acidentes de trânsito, estimando sua prevalência antes e após a vigência da "Lei Seca". Foram analisados 1.531 prontuários relativos aos acidentes de trânsito ocorridos no ano de 2007 e 2009. Houve prevalência de adultos jovens do sexo masculino, vítimas em sua maioria de atropelamento e acidentes envolvendo motos nos dois períodos estudados. Sobre o impacto da "Lei Seca" não foi observada grande redução no número de vítimas de acidentes de trânsito no período de estudo. Contudo, salienta-se sobre a importância da Lei em caráter permanente, pois esta é uma forte ferramenta para a prevenção de acidentes de trânsito no país.

Palavras-chave: Morbidade. Acidentes de trânsito. Emergências.

\begin{abstract}
It's a quantitative and descriptive study. The goals were designated to determine the number of subjects, victims of traffic accidents attended at an emergency department of a Municipal Hospital in Rio de Janeiro, before and after implementation of the "Prohibition"(Lei Seca), and identify the profile of this population and the characteristics of traffic accidents, estimating its prevalence before and after the term of "Prohibition" (Lei Seca). There were analyzed 1531 records relating to traffic accidents occurred in 2007 and 2009. There was a prevalence of young adult male, mostly victims of hitand-accidents involving motorcycles in both periods of study mentioned above. Concerning the impact of "Prohibition" (Lei Seca) was not observed a significant reduction in the number of victims on traffic accidents during the study period. However, there's no question about the importance of the law on a permanent basis, as this is a strong tool for the prevention of traffic accidents in the country.
\end{abstract}

Keywords: Morbidity. Accidents, Traffic. Emergencies.

\section{Resumen}

Estudio cuantitativo descriptivo. Los objetivos fueron determinar el número de individuos víctimas de accidentes de tránsito atendidos en un sector de urgencias de un Hospital Municipal de Rio de Janeiro antes y después de la implementación de la "Ley Seca" e identificar el perfil de esa población y las características de esos accidentes, estimando su prevalencia anterior y posterior a la vigencia de la "Ley Seca". Fueron analizados 1.531 prontuarios relativos a los accidentes de tránsito ocurridos entre los años 2007 y 2009. Hubo prevalencia de adultos jóvenes del sexo masculino, en su mayoría víctimas de atropellamiento y accidentes envolviendo motocicletas en los dos periodos analizados. Sobre el impacto de la "Ley Seca" no fue observada una significativa reducción en el número de víctimas de accidentes de tránsito en el referido periodo de estudio. Sin embargo, se sobresale la importancia de esta ley en carácter permanente, puesto que esta es una fuer te herramienta para la prevención de accidentes de tránsito en el país.

Palabras clave: Morbilidad. Accidentes de Tránsito.Urgencias Médicas

\footnotetext{
${ }^{1}$ Acadêmica de Enfermagem da Escola de Enfermagem Anna Nery/ Universidade Federal do Rio de Janeiro. Departamento de Enfermagem em Saúde Pública. Rio de Janeiro-RJ. Brasil. E-mail: analuap@msn.com; ²Professor Adjunto da Universidade Federal do Rio de Janeiro Departamento de Enfermagem em Saúde Pública. Escola de Enfermagem Anna Nery. Rio de Janeiro - RJ. Brasil E-mail:angelabreu@globo.com; ${ }^{3}$ Mestranda do Programa de Pós-Graduação em Enfermagem da Universidade Federal do Rio de Janeiro. Departamento de Enfermagem em Saúde Pública. Rio de Janeiro-RJ. Brasil. E-mail: louiseppaixao@gmail.com; ${ }^{4}$ Acadêmica de Enfermagem da Escola de Enfermagem Anna Nery/ Universidade Federal do Rio de Janeiro. Departamento de Enfermagem em Saúde Pública. Rio de Janeiro-RJ. Brasil. E-mail: vani_faria@yahoo.com.br
} 


\section{INTRODUÇÃO}

O consumo do álcool é um problema de saúde pública em todo o mundo, o uso indevido e o abuso da bebida alcoólica tornaram-se uma questão de saúde pública, trazendo consequências para 0 indivíduo, para a família e para a sociedade ${ }^{1-2}$

Nesse sentido, estudos nacionais e internacionais apontam o aumento do consumo de álcool em faixas etárias cada vez mais precoces ${ }^{3-4}$. Os jovens geralmente consomem mais bebidas alcoólicas e drogas do que os adultos, bem como tendem a desrespeitar normas de segurança no trânsito, 0 que, sabidamente, aumenta as chances de ocorrências de acidentes ${ }^{4}$.

Um estudo nacional demonstrou que, no período de 1990 a 2005, houve aumento de $72 \%$ de vítimas fatais de acidentes de trânsito em municípios com menos de 100 mil habitantes ${ }^{2}$. Ressalta-se que aproximadamente $70 \%$ dos acidentes violentos com mortes, no trânsito, o álcool é 0 principal responsável$\left.\right|^{5}$. No entanto, ainda que estudos venham apontando essa relação, pouco se tem estudado, no Brasil, a ocorrência do acidente e o nível de alcoolemia da vítima no momento do acidente ${ }^{3,5-6}$.

Diante desse contexto, o Congresso Nacional decretou a mais importante medida relacionada ao trânsito desde a criação do novo código de trânsito de 1997, a Lei 11.705, em 19 de Junho de 2008, popularmente conhecida como "Lei Seca". Essa Lei estabelece alcoolemia zero para os condutores de veículos; isto é, quem dirige não bebe, e quem insistir em beber e dirigir será multado, a carteira será apreendida, e o carro será retido até a apresentação de outro condutor habilitado $^{7-8}$.

Nesse sentido, já se pode observar uma redução de $23 \%$ no total de internações e de $22,5 \%$ das mortes por acidentes de trânsito ${ }^{2,7}$. Desse modo, o governo do Estado do Rio de Janeiro decidiu deflagrar, a partir de 19 de março de 2009, a "Operação Lei Seca”, como uma política pública, de caráter permanente no sentido de uma efetiva fiscalização no trânsito em todo o Estado e principalmente na cidade do Rio de janeiro. ${ }^{7-8}$.

Sendo assim, esse estudo traz os seguintes objetivos: determinar o número de indivíduos vítimas de acidentes de trânsito atendidos em um setor de emergência de um hospital municipal do Rio de Janeiro antes e depois da implementação da "Lei Seca"; identificar essa população e as características dos acidentes de trânsito, associadas a sua prevalência antes e após a vigência da "Lei Seca".

\section{MÉTODO}

Estudo com abordagem epidemiológica descritiva, realizado na emergência de um hospital municipal localizado na zona norte da cidade do Rio de Janeiro, cuja Área de
Planejamento (AP) é 3.2, de acordo com o planejamento sanitário de regionalização da cidade nas ações e serviços, da Secretaria Municipal de Saúde do Rio de Janeiro"; este hospital é considerado referência no atendimento de emergência para os seguintes bairros: Abolição, Água Santa, Cachambi, Del Castilho, Encantado, Engenho da Rainha, Engenho de Dentro, Engenho Novo, Higienópolis, Inhaúma, Jacarezinho, Jacaré, Lins de Vasconcelos, Maria da Graça, Méier, Piedade, Pilares, Riachuelo, Rocha, Sampaio, São Francisco Xavier, Todos os Santos e Tomás Coelho.

Foram analisados 5.327 prontuários, por todas as causas, no setor de documentação médica do hospital no período de janeiro, fevereiro e dezembro de 2007 antes da Lei e no mesmo período no ano de 2009 após a Lei, por meio de consulta aos prontuários dos pacientes atendidos na emergência independente do local de residência.

A escolha dos meses justifica-se por serem períodos comemorativos, como Ano Novo, Carnaval e Natal, e os períodos de 2007 e 2009 foram escolhidos pela ausência e presença da "LeiSeca", respectivamente. A população do estudo foi composta por 1.541 vítimas de acidentes de transito, correspondendo a $28,9 \%$ da população atendida na emergência, por todas as causas, no referido período.

Utilizaram-se como critério de inclusão os prontuários das vítimas de acidentes, cujo relato trazia referências a acidentes de trânsito, que deram entrada no serviço de emergência, nos meses estabelecidos. Foi considerado como limitação do estudo fonte de dados secundários; portanto, foram excluídos 10 prontuários preenchidos com letras ilegíveis, faltando dados no preenchimento, embora caracterizando de alguma forma 0 acidente de trânsito. Assim, chegou-se a amostra de conveniência constituída de 1.531 vítimas de acidentes.

As variáveis independentes foram relacionadas ao sexo, faixa etária e bairro de residência e as características do acidente (dias da semana, local do acidente, tipo de veículo e tipo de acidente).

Adotou-se, para identificação do bairro da vítima, a divisão administrativa por Áreas de Planejamento (AP), utilizada pelo setor de saúde para o município do Rio de Janeiro. Além dessa classificação, foram considerados Baixada Fluminense, Niterói e adjacências.

Os dados foram processados eletronicamente por meio do programa EPI-Info versão 3.5.1. As análises bivariadas foram feitas a partir de estatísticas descritivas e apresentadas em tabelas. 0 teste do qui-quadrado de Pearson foi utilizado para avaliação de associação entre variáveis categóricas, sendo adotado o nível de $5 \%$ para significância estatística.

0 estudo foi submetido aos procedimentos normativos do Comitê de Ética e Pesquisa da Secretaria Municipal de Saúde e Defesa Civil do Rio de Janeiro (SMSDC), sob o protocolo n $55 / 10$. 


\section{RESULTADOS}

Na Tabela 1, a seguir, observou-se que, de um total de 5.327 atendimentos realizados por todas as causas na emergência do Hospital Municipal Salgado Filho, durante os meses de janeiro, fevereiro e dezembro de 2007 e os mesmos meses em 2009, $2.386(44,7 \%)$ atendimentos ocorreram no ano de 2007 e 2.941 $(55,2 \%)$ atendimentos ocorreram no ano de 2009. Desses, 1.531
$(28,7 \%)$ foram atendimentos realizados às vítimas de trânsito; observou-se que 706 (46,1\%) ocorreram no ano de 2007, antes da "LeiSeca", e 825 (53,8\%) ocorreram no ano de 2009, após a "Lei Seca". Os resultados mostraram uma associação estatisticamente significativa quando se compara o total de atendimentos por todas as causas com o total de atendimentos às vítimas de acidentes de trânsito nos períodos relativos aos anos de 2007 e 2009, antes e após a "Lei Seca" ( $p<0,001$ ).

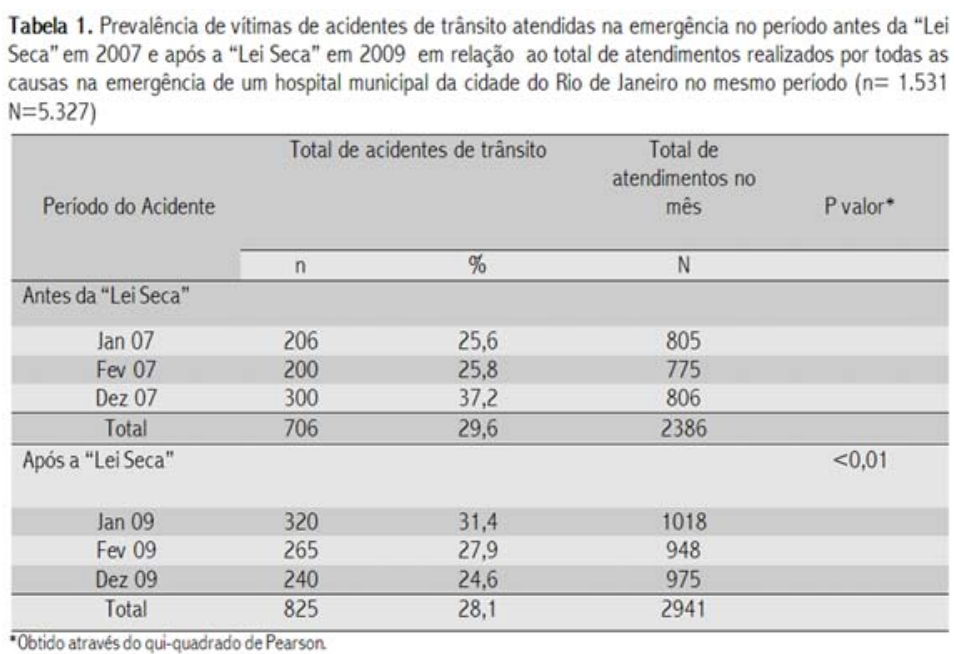

Observamos que entre as 1.531 vítimas de acidentes de trânsito atendidas na emergência, a maioria pertencia ao sexo masculino no anos de 2007 (73,7\%) e 2009 (69,9\%). No que se refere à faixa etária, houve predominância de vítimas com idades entre 21 a 40 anos (50,9\% em 2007 e 54,4\% em 2009). Em relação à Área de Planejamento (A.P.), observou- se que 635 vítimas de trânsito eram provenientes da A.P. 3.2 (44,5\% do ano de 2007 e $38,9 \%$ do ano de 2009 ), seguidas de 314 vítimas atendidas da A.P. 3.3 (17,4\% em 2007 e $23,2 \%$ em 2009); 134 vítimas tiveram a Área de Planejamento ignorada, correspondendo a 10,5\% em 2007 e 7,3\% em 2009 (Tabela 2).

Tabela 2. Distribuição por sexo, faixa etária e bairro de residência das vitimas atendidas em uma emergência de um hospital municipal da cidade do Rio de Janeiro, associada à frequência dos acidentes de trânsito antes da "Lei Seca" (2007 - $n=706)$ e após a "Lei Seca" (2009 - $n=825)$. $n=1531$

\begin{tabular}{|c|c|c|c|c|c|}
\hline \multirow[t]{2}{*}{ Variáveis } & \multicolumn{2}{|c|}{ Antes "Lei Seca" (2007) } & \multicolumn{2}{|c|}{ Após "Lei Seca" (2009) } & \multirow[b]{2}{*}{ Valor $p$} \\
\hline & $n$ & $\%$ & $n$ & $\%$ & \\
\hline Sexo & & & & & 0,55 \\
\hline Feminino & 186 & 26,3 & 248 & 30,1 & \\
\hline Masculino & 520 & 73,7 & 577 & 69,9 & \\
\hline Faixa etária & & & & & 0,24 \\
\hline$<10$ anos & 42 & 6,0 & 52 & 6,4 & \\
\hline 11 a 20 anos & 119 & 17,0 & 105 & 12,9 & \\
\hline 21 a 40 anos & 356 & 50,9 & 443 & 54,4 & \\
\hline 41 a 60 anos & 117 & 16,7 & 135 & 16,6 & \\
\hline$>61$ anos & 65 & 9,3 & 80 & 9,8 & \\
\hline Bairro de residência & & & & & 0,00 \\
\hline A.P. 1.0 & 35 & 5,0 & 26 & 3,2 & \\
\hline A.P. 2.1 & 03 & 0,4 & 04 & 0,5 & \\
\hline A.P. 2.2 & 12 & 1,7 & 05 & 0,6 & \\
\hline A.P. 3.1 & 47 & 6,7 & 53 & 6,5 & \\
\hline A.P. $3.2^{*}$ & 314 & 44,5 & 321 & 38,9 & \\
\hline A.P. $3.3 \cdots$ & 123 & 17,4 & 191 & 23,2 & \\
\hline A.P. 4.0 & 32 & 4,5 & 77 & 9,3 & \\
\hline AP. 5.1 & 24 & 3,4 & 35 & 4,2 & \\
\hline A.P. 5.2 & 06 & 0,8 & 02 & 0,2 & \\
\hline A.P. 5.3 & 06 & 0,8 & 06 & 0,7 & \\
\hline Baixada Fluminense & 24 & 3,4 & 42 & 5,1 & \\
\hline Niterói e adjacências & 06 & 0,8 & 02 & 0,2 & \\
\hline Ignorado & 74 & 10,5 & 60 & 7,3 & \\
\hline
\end{tabular}

-AP 3.2: Abolição, Aqua Santa, Cachambi, Del Castiho, Encantado, Engenho da Rainha, Engenho de Dentro, Engenho Nover, Higienópols, Inhaúma, Jacarezinho, Jacaré, Lins de Vasconcelos, Maria da Graça, Méier, Piedade, Pliares, Rlachuelo,Pocha, Sampaio, São Francisco Xavier, Todos os Santos e Tomás Coeho.

- AP 3.3: Acari, Anchieta, Barros Filhos, Bento Roeiro, Campinho, Cascadura, Cavakante, Coeho Neto, Costa Barros, Engenheiro Leal, Guadalupe, Honório Gurgel, Irajä, Madureira, Marechal Hermes, Oswaldo Cruz, Pavuna, Quintino, Bocaiuva, Ricardo de Abuquerque, Rocha Miranda, Turiaçu, Vaz Lobo, Vicente de Carvaho, Via da Penha, Vista Alegre e Parque Anchieta 
Na Tabela 3, podemos observar que, ao se tratar das características dos acidentes de trânsito das vítimas atendidas na emergência, em relação ao dia da semana, identificou-se predominância de finais de semana, principalmente aos sábados, totalizando 282 vítimas (18,8\% em 2007 e 18,1\% em 2009), e domingos, apresentando 274 (16,7\% em 2007 e 18,9\% em 2009).

\begin{tabular}{|c|c|c|c|c|c|}
\hline \multirow{2}{*}{ Variáveis } & \multicolumn{2}{|c|}{ Periodo antes da "Lei Seca" } & \multicolumn{2}{|c|}{ Periodo após a "Lei Seca" } & \multirow{2}{*}{ Valor $p$} \\
\hline & $n$ & $\%$ & $n$ & $\%$ & \\
\hline \multicolumn{6}{|l|}{ Dias da Semana } \\
\hline & & & & & 0,08 \\
\hline Domingo & 118 & 16,7 & 156 & 18,9 & \\
\hline Segunda & 117 & 16,6 & 97 & 11,8 & \\
\hline Terça & 95 & 13,5 & 112 & 13,6 & \\
\hline Quarta & 81 & 11,5 & 96 & 11,6 & \\
\hline Quinta & 69 & 9,8 & 108 & 13,1 & \\
\hline Sexta & 93 & 13,2 & 107 & 13,0 & \\
\hline Sábado & 133 & 18,8 & 149 & 18,1 & \\
\hline \multicolumn{6}{|l|}{ Tipo de veiculo } \\
\hline Automóvel & 156 & 22,1 & 193 & 23,4 & \multirow[t]{7}{*}{0,00} \\
\hline Bicicleta & 12 & 1,7 & 11 & 1,3 & \\
\hline Caminhão & 06 & 0,8 & 05 & 0,6 & \\
\hline Moto & 284 & 40,2 & 349 & 42,3 & \\
\hline Onibus & 34 & 4,8 & 79 & 9,6 & \\
\hline Outros & 13 & 1,8 & 24 & 2,9 & \\
\hline Ignorado & 201 & 28,5 & 164 & 19,9 & \\
\hline \multicolumn{6}{|l|}{ Tipo de acidente } \\
\hline Atropelamento & 240 & 34,0 & 244 & 29,6 & \multirow{6}{*}{0,00} \\
\hline Colisão & 145 & 20,5 & 238 & 28,8 & \\
\hline Queda de bicicleta & 02 & 0,3 & 01 & 0,1 & \\
\hline Queda de moto & 201 & 28,5 & 229 & 27,8 & \\
\hline Outros & 24 & 3,4 & 36 & 4,4 & \\
\hline Ignorado & 94 & 13,3 & 77 & 9,3 & \\
\hline
\end{tabular}

No que se refere ao tipo de veículo da vítima, houve a prevalência dos acidentes envolvendo motos com 633 vítimas (40,2\% em 2007 e 42,3\% em 2009), seguido do automóvel com 349 vítimas (22,1\% em 2007 e 23,4\% em 2009). Ao tratar do tipo de acidente, 0 atropelamento foi predominante, com 484 vítimas (34,0\% em 2007 e 29,6\% em 2009), seguido da queda de moto com 430 vítimas (28,5\% em 2007 e 27,8\% em 2009) e da colisão, com 383 vítimas (20,5\% em 2007 e 28,8\% em 2009) (Tabela 3).

\section{DISCUSSÃO}

0 sexo masculino e a faixa etária jovem, economicamente ativa, foram predominantes nesse estudo, representaram a maioria das vítimas, o que vai ao encontro de muitas pesquisas que abordam essa temática. ${ }^{2,3,5,10}$ Esses dados ainda ressaltam o possível impacto e prejuízos econômicos sobre esse seguimento da população, influenciando no indicador Anos Potenciais de Vida Perdidos, visto que as vítimas de trânsito são principalmente da população jovem ${ }^{1,9,11-12}$.

Vê-se a importante diferença entre os sexos no que tange à frequência do atendimento por esses tipos de acidentes.
Esses resultados podem ser explicados, em parte, pelo comportamento da população masculina, podendo ser atribuído à sua maior exposição, dado que os homens têm comportamentos sociais e culturais que os predispõem, em maiores proporções, aos riscos de lesões e mortes: direção em maior velocidade, maior consumo de álcool, maior agressividade, tendência a realizar manobras arriscadas, dentre outros ${ }^{1,12-13}$.

No que diz respeito ao período do acidente das vítimas de trânsito atendidas na emergência, chama-nos atenção 0 período de janeiro de 2009, quando observamos o maior índice de acidentes no total, ou seja, superou o ano de 2007 nos meses de janeiro e fevereiro mesmo após a implementação da Lei da alcoolemia zero. No entanto, quando comparamos os meses de dezembro de 2007 e dezembro de 2009, observamos uma diminuição dos acidentes em 2009, o que podemos atribuir à implantação, pelo governo de Estado, da fiscalização denominada "Operação Lei Seca", que passou a acontecer na cidade do Rio de Janeiro efetivamente em março de 20097-8, 12 .

Sendo assim, pesquisas adicionais devem ser realizadas para verificar o efetivo impacto da "Lei Seca" no atendimento das unidades de emergências dos hospitais do Rio de Janeiro. No entanto, cabe ressaltar que a fiscalização no trânsito, por 
meio da Operação "Lei Seca", é uma das ferramentas importantes utilizadas no sentido de colaborar com as políticas publicas de prevenção, diminuindo a assustadora estatística de morbimortalidade por acidentes de trânsito na grande área metropolitana do Rio de Janeiro diariamente, conforme aponta alguns estudos 5,7-9,12.

Observou-se maior ocorrência de acidentes de trânsito nos finais de semana, concentrando-se em sábados e domingos. Esse achado vem ao encontro de outros estudos que apontam essa concentração de acidentes principalmente nos fins de semana ${ }^{1,5,11-12}$. Esses dados podem sugerir que nos finais de semana as pessoas tendem a abusar um pouco mais do consumo da bebida alcoólica, o que, consequentemente, pode levar a um nível de risco maior, ou maior frequência de acidentes provocados pelo binômio álcool e trânsito em todos os sentidos, tanto para os pedestres quanto para os motoristas ${ }^{1,7,11-12}$.

Percebe-se, em seguida, uma maior frequência dos acidentes na A.P. 3.2 nos dois períodos estudados, o que era de se esperar, visto que a localização do hospital se insere nesta área programática, e, quando comparado a 2009, percebe-se um aumento considerável desses acidentes nessa área.

Ressaltamos aqui, por conta desses dados encontrados, a importância de chamar atenção para alguns estudos que relatam que os acidentes de trânsito são responsáveis pelos principais atendimentos nas grandes emergências dos hospitais urbanos e, ainda, para o fato de que quanto maior a gravidade do acidente maior é o seu envolvimento com bebidas alcoólicas 1, 5, 7, 11-12

Dessa forma, ressaltamos que, em 2009, já havia sido implantada a Lei de Alcoolemia Zero, em nível nacional; no entanto, em dezembro do referido ano, a fiscalização intitulada "Operação Lei Seca" estava efetivamente implementada na cidade do Rio de janeiro ${ }^{8,12}$ e atuando principalmente aos finais de semana e, mesmo assim, nos deparamos com um certo aumento do número de vítimas nessa região e em finais de semana.

0 presente estudo identificou o atropelamento como 0 tipo de acidente com maior frequência nos dois períodos, e, quanto ao tipo de veículos, encontramos a moto como fator preponderante nesses acidentes.

No Brasil, $19 \%$ das mortes em acidentes de trânsito estão relacionadas ao atropelamento ${ }^{13-14}$, o que caracteriza um país subdesenvolvido, nesse cenário, refletindo cidades que crescem muito e de forma desordenada ${ }^{1,11,13-15}$. Nesse sentido, campanhas educativas têm sido apontadas como componente essencial para a prevenção das lesões entre pedestres, com ênfase especial para os grupos de crianças e idosos, sendo esta faixa etária a mais acometida ${ }^{14}$. Isso nos chama atenção para o resultado de nosso estudo, que apontou um percentual significativo de vítimas por acidentes de trânsito menores de 10 anos e maiores de 61 anos, nos dois períodos estudados, em atendimento na emergência, conforme mostra Tabela 2.
Nos últimos anos observou-se aumento no risco de morte por acidente envolvendo motos ${ }^{10}$. De acordo com o Painel de Indicadores do SUS, a mortalidade por acidente com motocicleta continua crescendo em todo o país. Em 2006, nas regiões Centro-Oeste e Nordeste, o óbito por acidente com moto, pela primeira vez, superou o óbito por acidente de automóvel ${ }^{15}$.

Contudo, vale ressaltar que o número de acidentes envolvendo motos vem aumentando nos atendimentos das emergências nos últimos anos nas grandes cidades do país de uma forma geral, vindo ao encontro dos dados encontrados para esse tipo de veiculo neste estudo, apontando um aumento significativo quando comparado aos acidentes com automóveis 1,7 .

0 crescente processo de urbanização tem proporcionado que motocicletas sejam amplamente utilizadas como meio de transporte. A rapidez do veículo, a mobilidade, o baixo custo, e até mesmo a utilização como instrumento de trabalho, como de "motoboys" e "mototaxistas", levaram a um cres-cimento exponencial no número de motocicletas circulantes ${ }^{11,15}$.

\section{CONSIDERAÇÕES FINAIS}

0 presente estudo utilizou fonte de dados secundários por meio de registros do próprio Serviço de Saúde, embora com limitações atribuídas a grandes frequências de dados ignorados e um levantamento realizado em apenas três meses, embora em dois períodos relevantes, antes e após a "LeiSeca". Os dados alertam sobre a relevância de uma fiscalização direta, efetiva e contínua, levando-se em consideração a insuficiência de medidas preventivas para os acidentes de trânsito.

No entanto, estudos sobre o tema são de notória importância, principalmente se for considerado que as estatísticas disponíveis no país, em relação ao assunto, ainda são insuficientes, não demonstrando a real face da situação. Percebe-se o elevado índice de casos de acidentes de trânsito atendidos no setor de emergência, quando comparados a todas as causas. Nota-se ainda uma frequência elevada de acidentes de trânsito envolvendo motociclistas. A vulnerabilidade do motociclista, em particular, tem sido demonstrada pelo elevado número de acidentes envolvendo essa populaçãa 1,5,7,11-12.

Dessa forma, deve-se refletir sobre a importância de políticas públicas de prevenção de acidentes de trânsito, e sobre a sua aplicabilidade para que se tenha uma vida no trânsito mais segura. Faz-se necessária também a conscientização da população diante dessas políticas, para que elas possam se perpetuar no quesito viabilidade.

Logo, ressaltamos a importância de continuarmos nosso papel como profissionais da saúde, informando, orientando, sobretudo educando, em relação à segurança no trânsito relacionada ao binômio álcool e direção, indo ao 
encontro das atuais políticas públicas nessa temática, lembrando que estamos em plena década de "Aç̃̃es para Segurança Rodoviária" no mundo, estabelecidas pela Organização das Nações Unidas/ ONU (2011-2020) a fim de traçar e direcionar melhor metas para conter o crescimento dos acidentes de trânsito, sobretudo nos países em desenvolvimento.

\section{REFERÊNCIAS}

1.Abreu, AMM, Lima JMB, Alves TA. 0 Impacto do Álcool na Mortalidade em Acidentes de Trânsito: uma questão de Saúde Pública. Esc Anna Nery. $2006 \mathrm{abr} ; 10(1): 87-94$.

2.Malta DC et.al, Nota Técnica: Impacto da Legislação Restritiva do Álcool na Morbimortalidade por Acidentes de Transporte Terrestre - Brasil. Epidemiol. serv. saúde. 2010 mar; 19(1): 77-8.

3.Secretaria Nacional Antidrogas (SENAD), Universidade Federal de São Paulo (UNIFESP). $1^{\circ}$ Levantamento Nacional sobre os Padrões de Consumo de Álcool na População Brasileira. Brasília(DF): [Profissionais do Texto]; 2007.

4.Lima JMB. Alcoologia: O Alcoolismo na Perspectiva da Saúde Pública. Rio de Janeiro(RJ): Medbook; 2012.

5.Abreu AMM, Lima JMB, Matos LN, Pillon SC. Uso de Álcool em Vítimas de Acidentes de Trânsito: Estudo do Nível de Alcoolemia. Rev. Latino-Am. Enfermagem. 2010 mai/jun; 18(3): 513-20.

6.Orosco R, Borges G, Mondragón L, Monroy-Nasr Z. El lugar donde ocurren lãs lesiones y su relacion com el uso de alcohol. Estúdio em sala de urgências. Salud ment. 2005 out; 28(5): 50-6.

7.Moreira F. A mudança cultural que salva vidas: a lei que salva vidas e a "vacina" contra a violência do trânsito. Rio de Janeiro(RJ): Arquimedes edições; 2008.

8.Lopes CA. Operação LeiSeca: nunca dirija após beber. Rio de Janeiro(R)): [sem editor]; 2010. [folder]

9.Ministério da Saúde (BR). $4^{\circ}$ Seminário de Gestão Participativa da Região Metropolitana, Fórum de Conselhos Municipais de Saúde da Região Metropolitana I-Reorganizando o SUS no Rio de Janeiro, 2004 dez 16; Rio de Janeiro, Brasil. Brasília(DF): MS Editora; 2005.

10.Abreu AMM, Lima JMB, Griep RH. Acidentes de Trânsito e a Frequência dos Exames de Alcoolemia com Vítimas Fatais na Cidade do Rio de Janeiro. Esc Anna Nery. 2009 jan/mar; 13(1): 44-50.

11.Filho AM. Motocicletas: 0 Conflito entre Agilidade e Segurança. Rev Abramet. 2007; 48: 23-7.

12.Abreu AMM, Jomar RT, Thomaz RGF, Guimarães RM, Lima JMBL, Figueiró RFS. Impacto da Lei Seca na Mor talidade por Acidentes de Trânsito. Rev. enferm. UERJ. $2012 \mathrm{jan} / \mathrm{mar} ; 20(1): 21-6$

13.Lima MADS, Pereira WAP. Atendimento Pré-hospitalar: Caracterização das Ocorrências de Acidentes de Trânsito. Acta paul. enferm. 2006 jul/set; 19(3): 279-83.

14.Gawryszewski VP, Coelho HMM, Scarpelini S, Zan R, Jorge MHPM, Rodrigues SEM. Perfil dos atendimentos a Acidentes de Transporte Terrestre por Serviços de Emergência em São Paulo. 2005. Rev. saúde pública. 2009 abr; 43(2): 275-82.

15.Ministério da Saúde (BR), Secretaria de gestão estratégica e participativa, Departamento de monitoramento e avaliação de gestão do SUS. Panorâmico. Painel panorâmico de Indicadores do SUS. [Sem data]; 3(7): 1-84 